\title{
Equivalence of the generalised grid and projection methods for the construction of quasiperiodic tilings
}

\author{
F Gähler and J Rhyner \\ Theoretische Physik, Eidgenössische Technische Hochschule, CH-8093 Zürich, Swizerland
}

Received 16 August 1985

\begin{abstract}
The two main techniques for the generation of quasiperiodic tilings, de Bruijn's grid method and the projection formalism, are generalised. A very broad class of quasiperiodic tilings is obtained in this way. The two generalised methods are shown to be equivalent. The standard calculation of Fourier spectra is extended to the whole general class of tilings. Various applications are discussed.
\end{abstract}

\section{Introduction}

The recent discovery of a new phase of Al-Mn alloy (Shechtman et al 1984) exhibiting icosahedrally symmetric diffraction patterns has led physicists " interest to quasiperiodic tilings. Prototypes of quasiperiodic tilings are the famous Penrose patterns (Gardner 1977, Penrose 1979). For these, a delailed algebraic theory had been developed by de Bruijn (1981). He showed that these tilings can be constructed from the so-called 'pentagrid'. This construction has a nice geometric interpretation: a Penrose pattem can be viewed as the projection of a surface in a SD cubic lattice onto a suitable plane.

de Bruijn's ideas were soon generalised by Kramer and Neri (1984). In their prophetic work, published well before Shechtman's discovery, they constructed 3D icosahedrally symmetric tilings by projecting a $3 \mathrm{D}$ lattice hypersurface in a $6 \mathrm{D}$ cubic lattice onto a suitable 3-space. These tilings are composed of the two rhombohedra discussed earlier by Mackay (1981).

After a first attempt by Levine and Steinhardt (1984) along slightly different lines, several authors (Elser 1985, Duneau and Katz 1985, Kalugin et al 1985) interpreted the results of Shechtman et al in the spirit of Kramer and Neri. All these authors made use of the fact that the projection formalism offers an ingenious way of calculating the Fourier spectra analytically. Also other structural properties are studied most conveniently in the projection framework. For instance, Elser (1985) was able to calculate the frequency of occurrence of any given finite subpattern. On the other hand, the grid technique is best suited for the construction of tilings on a computer, yielding an algorithm that is much more transparent than a direct application of the projection method.

In this paper we introduce generalisations of both the grid and the projection technique. In this way we are able to construct a broad class of quasiperiodic tilings, e.g. such with any desired point symmetry. For the projection method, similar proposals have been made already by Duneau and Katz (1985). The main point is then to prove the equivalence of the two generalised methods. Our proof is a constructive one. It thus allows us to switch from one scheme to another depending on which one is more 
convenient for the purpose. In this way, various techniques originally developed for one method also become available for the other. We believe that this will provide new and powerful tools for the study of quasiperiodic tilings.

This paper is organised as follows. After briefly reviewing the 'classical' grid and projection methods, we introduce our generalisations. Then the equivalence of the two generalised methods is proven. Using this proof, in $\$ 5$ the projection technique of calculating Fourier spectra is extended to the whole general class of quasiperiodic patterns constructed earlier. We close with two examples. First we discuss 2D tilings with $n$-fold point symmetry. Then a new principle is presented for the construction of quasiperiodic tilings containing large periodic regions.

\section{2. de Bruijn's grid method}

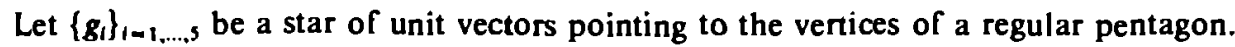
We call them grid vectors. The pentagrid (or simply grid) $G_{5}$ is defined as the union of five arrays of equidistant parallel lines orthogon $I$ to the vectors $g_{i}$ :

$$
G_{5}=\left\{x \in E^{2} \mid x \cdot g_{l}-\gamma_{1}=k_{l} ; l=1, \ldots, 5, k_{l} \in \mathbb{Z}\right\} .
$$

The grid parameters $\gamma_{1} \in \mathbf{R}$ are translations of the arrays relative to the origint. We assume the $\gamma_{l}$ to be such that nowhere do more than two grid lines intersect. Such grids are called regular.

To every point $y \in E^{2}$ we assign a vector $K(y) \in Z^{5}$ through

$$
K_{i}(y)=\left\lceil y \cdot g_{1}-\gamma_{1}\right\rceil \quad i=1, \ldots, 5
$$

where $[x]=\min \{n \in Z \mid n \geqslant x\}$. Let $x_{0}$ be an intersection point of two grid lines and $U\left(x_{0}\right)$ a small neighbourhood of $x_{0}$ containing no other intersection points. From (1) and (2), it follows that on $U\left(x_{0}\right)$ the vector function $K(y)$ takes on four different values $K\left(x_{0}, j\right), j=1, \ldots, 4$. One readily notes that the four vectors

$$
P\left(x_{0}, j\right)=\sum_{i=1}^{5} K_{i}\left(x_{0, j} j\right) \cdot g_{i} \quad j=1, \ldots, 4
$$

point to the vertices of one of the two Penrose rhombuses. The set of rhombuses defined by all intersection points of the grid form a perfect tiling (de Bruijn 1981), i.e. one with no holes or overlaps.

\section{The projection method}

In this section we review the projection method as discussed by Duneau and Katz (1985). For simplicity, we first restrict ourselves to cubic lattices. More general ones will be included later in this paper.

Let $\mathscr{L}$ be the union of $N$ arrays of hyperplanes in $E^{N}$ :

$$
\mathscr{L}=\left\{x \in E^{N} \mid x \cdot h_{1}-y_{l}=k_{l} ; l=1, \ldots, N, k_{1} \in Z\right\}
$$

where $\left\{h_{1}\right\}_{1-1, \ldots, N}$ is an orthonormal basis of $E^{N}$. It will turn out that the $\gamma_{i}$ occurring in (4) can in fact be identified with the grid parameters introduced in the last section. 
The points where $N$ hyperplanes intersect are the vertices of a simple cubic lattice $L$ given by

$$
L=\left\{x \in E^{N} \mid x=\sum_{l=1}^{N}\left(\gamma_{l}+k_{l}\right) \cdot h_{h} k_{l} \in Z\right\} .
$$

Clearly, the $\gamma_{1}$ are the coordinates of the origin of $L$ Let $E^{D}$ be a $D$-dimensional subspace of $E^{N}$. We define an open strip $S$ around $E^{D}$ by

$$
\left.\left.S=\left\{x=x_{1}+\sum_{i=1}^{N} \alpha_{i} h_{h, 1} \mid x_{1} \in E^{D}, \alpha_{i} \in\right]-1,0\right] \forall i\right\}
$$

where $h_{i 1}$ is the component of $h_{i}$ orthogonal to $E^{D}$. In other words, $S$ is generated by moving a unit cube along $E^{D}$ in such a way that its corner with the highest coordinates is always contained in $E^{D}$.

From the construction of $S$ it follows that for generic $\gamma$ there is a unique $D$. dimensional lattice hypersurface $\Sigma$ contained in $S$. The vertices of $\Sigma$ are the corners with the lowest coordinates of those unit hypercubes of the lattice which have non-zero intersection with $E^{D}$. The tiling is then the orthogonal projection of $\Sigma$ onto $E^{D}$. It is composed of the projections of the $\left(\begin{array}{c}N \\ D\end{array}\right)$ different unit $D$ cells of the lattice.

The connection to the grid method consists in the following observations. The grid $G_{N}$ has to be identified with the intersection $\mathscr{L} \cap E^{D} \uparrow . \mathscr{L}$ divides $E^{D}$ into open regions, and with everyone of these we can associate a cube of the lattice intersecting with $E^{D}$. The projections of the corners with the lowest coordinates of these cubes can then be calculated. This is precisely what is done in the grid method.

de Bruijn's Penrose pattern constructed in the preceding section can be obtained by choosing $N=5, D=2$, and by properly embedding the subspace $E^{2}$ in $E^{3}$. For details see $\S 8$ or the original paper of de Bruijn (1981).

\section{The generalised grid method}

We extend the grid method along three lines.

(i) We construct tilings of any dimension $D$. For this purpose, we define a $D$-dimensional grid which is the union of $(D-1)$-dimensional hyperplanes in $E^{D}$.

(ii) The grid vectors $g_{i}$ can be any $N$ vectors in $E^{D}$ which together span $E^{D}$.

(iii) The vectors which span the tiles (the tiling vectors) need not to be identical to the grid vectors. With every grid vector $g_{i}$ we can associate an essentially freely chosen tiling vector $t_{i}$. The only restriction is that, for every $D$-tuple $\left(i_{1}, \ldots, i_{D}\right)$ of indices, the corresponding $D$-tuples of grid and tiling vectors, $\left(g_{1}, \ldots, g_{b_{D}}\right)$ and $\left(t_{i}, \ldots, t_{i p}\right)$, span a volume of the same orientation. From geometrical considerations in the next section it will become clear that this condition is necessary and sufficient for the tiling not to overlap. This is true at least for dimensions $D \leqslant 3$, and we conjecture that it holds for any $D$.

Following these three points, we define an $N$ grid $G_{N} \subset E^{D}$ by

$$
G_{N}=\left\{x \in E^{D}\left|x \cdot \hat{g}_{t}-y_{t}=k_{1}\right| g_{1} \mid ; l=1, \ldots, N, k_{l} \in Z\right\}
$$

where $\hat{g}_{l}=g_{1} /\left|g_{1}\right|$. The orthogonal distance between two neighbouring hyperplanes of t Note that our terminology diflers from that of Kramer and Neri (1984). They use the term 'grid' for $\mathscr{L}$. 
the th array is thus $\left|g_{1}\right|$. Again we assume the grid to be regular; there are no points where more than $D$ hyperplanes intersect. As before, we define a vector function $K: E^{D} \rightarrow \mathbb{Z}^{N}$ by its components:

$$
\left.K_{i}(y)=\left.|| g_{i}\right|^{-1} \cdot\left(y \cdot \hat{\boldsymbol{g}}_{i}-\gamma_{i}\right)\right\rceil \quad i=1, \ldots, N .
$$

On a small neighbourhood of an intersection point $x_{0}$ of $D$ grid hyperplanes, $K(y)$ takes $2^{D}$ different values $K\left(x_{0}, j\right), j=1, \ldots, 2^{D}$. The vectors

$$
P\left(x_{0}, j\right)=\sum_{i=1}^{N} K_{i}\left(x_{0}, j\right) \cdot t_{i} \quad j=1, \ldots, 2^{D}
$$

point to the vertices of a D.dimensional parallelepiped. We claim that the parallelepipeds corresponding to all intersection points of the grid form a perfect tiling of $E^{D}$, provided the "non-overlapping condition' is satisfied. This will follow from the correspondence of the above grid method with the generalised projection method presented in the next section.

\section{The generalised projection method}

The "classical' projection method is extended with respect 10 the following three points.

(i) The formalism easily applies to general, not necessarily cubic, lattices. If $\left\{h_{1}\right\}_{1-1, \ldots, N}$ is a basis in $E^{N}$, the definition corresponding to (4) is

$$
\mathscr{L}=\left\{\mathrm{r} \in E^{N}\left|x \cdot \hat{h_{1}}-\gamma_{l}=k_{I} \cdot\right| h_{1} \mid ; l=1, \ldots, N, k_{1} \in \mathbb{Z}\right\}
$$

where $\hat{h}_{h}=h_{h} /\left|h_{1}\right|$. The points where $N$ hyperplanes of $\mathscr{L}$ intersect form a lattice $L$ which is generated by that basis $\left\{b_{i}\right\}$ in $E^{N}$ which spans an elementary cell of $\mathscr{L}$. The strip $S$ around a $D$-dimensional subspace $E^{D}$ is still given by (6), but with $\left\{h_{1}\right\}$ replaced by $\left\{b_{i}\right\}$. For orthogonal lattices, $\left\{b_{l}\right\}$ is identical to $\left\{h_{i}\right\}$. Since $\mathscr{L} \cap E^{D}$ has to be identified with the grid $G_{N}$ we call $E^{D}$ the $g$ nid space and denote it by $E_{G}^{D}$.

(ii) The unique $D$-dimensional lattice hypersurface $\Sigma$ contained in $S$ is projected onto the tiling space $E_{T}^{D}$, a $D$-dimensional subspace of $E^{N}$ which is not necessarily parallel to $E_{G}^{b}$. The grid space and the tiling space thus have to be carefully distinguished,

(iii) We apply a general regular linear transformation $T: E_{T}^{D} \rightarrow E_{T}^{D}$ to the projected pattern. The tiling thus is obtained by applying a linear transformation $A$ to the hypersurface $\Sigma$, where $A$ is the product of the orthogonal projection $P$ onto $E_{T}^{D}$ and a subsequent linear transformation $T$ within $E_{T}^{D}$.

The first two points have already been mentioned as possible extensions by Duneau and Katz (1985).

Remark If we want to avoid overlapping tilings, we are not completely free any more in embedding the tiling space in $E^{N}$, once the lattice and the grid space are fixed. We have to embed $E_{T}^{D}$ such that the orthogonal projection $P$ of the lattice hypersurface $\Sigma$ onto $E_{T}^{D}$ is an injective mapping. This is the case if it is possible to choose orientations on the hypersurfaces $\Sigma$ and $E_{T}^{D}$ such that $P$ is (everywhere) orientation preserving. The generalised projection method and the non-overlapping condition are visualised in figure 1 for the simple case $N=2, D=1$. In figure $1(b)$ one sees that for either orientation on $\Sigma$ there are segments on $\Sigma$ where $\boldsymbol{P}$ changes the orientation. Bearing this geometrical situation in mind, the reader will easily verify that the non-overlapping condition stated for the generalised grid method is in fact the correct one. 
(a)

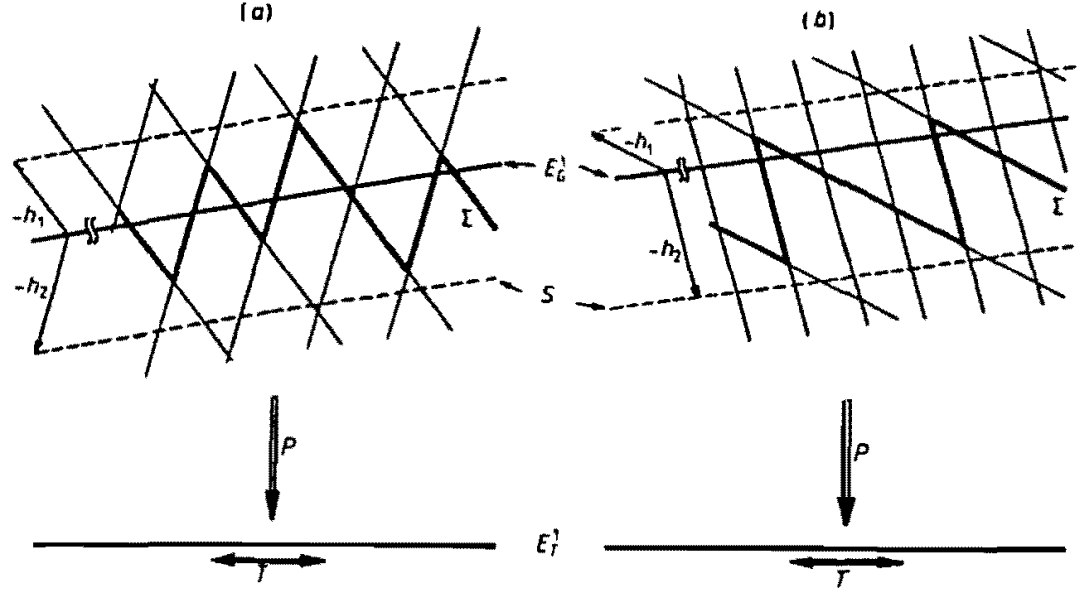

Figure I. Visualisation of the projection procedure for the simple case $N=2, D=1$. The non-overlapping condition is satisfed in $(a)$ and violated in $(b)$.

\section{The equivalence of the two generalised methods}

From the previous arguments it has become clear that every tiling constructed by the generalised projection technique can also be obtained by the generalised grid method. Recall that we have assumed a generic position of $E_{G}^{D}$, i.e. we assume that $\mathscr{L}_{\cap} E_{G}^{D}$ is a regular $N$ grid. The extension to singular $N$ grids and grid space positions is straightforward, see e.g. de Bruijn (1981). It remains to show that the converse is true as well.

Let us consider a fixed tiling, defined by a regular $N$ grid $G_{N}$ and a set of tiling vectors $\left\{t_{1}\right\}_{i=1, \ldots . N}$. We can always find a lattice $\mathscr{L}$ of hyperplanes $\dagger$

$$
\mathscr{L}=\left\{x \in E^{N}\left|x \cdot \hat{h}_{1}-\gamma_{l}=k_{1} \cdot\right| h_{1} \mid ; l=1, \ldots, N, k_{1} \in \mathbf{Z}\right\}
$$

such that the grid $G_{N}^{D}$ is just the intersection of $\mathscr{L}$ with some $D$-dimensional subspace of $E^{N}$. This subspace thus has' to be identified with the grid space $E_{G}^{D}$. In (11) we assume, of course, that the vectors $\left\{h_{i}\right\}_{i-1, \ldots, N}$ generating $\mathscr{L}$ are linearly independent.

Note that the vectors $h_{\text {, }}$ and the grid vectors $g$, have to be enumerated consistently. The vector $h_{l}$ points from one hyperplane of $\mathscr{L}$ to the next in the same array, and so it should be $g_{1}$ which connects the intersections of these two hyperplanes with the grid space $E_{G}^{D}$.

The strip $S$ around $E_{G}^{D}$, containing a unique $D$-dimensional lattice hypersurface $\Sigma$, is again given by (6), but with $h_{i}$ replaced by $b_{i}$. The main point is now to find the correct embedding of the tiling space, containing the tiling vectors, in $E^{N}$. (The tiling vectors $t_{i}$ are supposed to be fixed within $E_{T}^{D}$.) In general, we can neither hope that it is possible to choose the grid and the tiling spaces to be the same nor that the tiling vectors are the projections of the lattice vectors $b_{;}$onto the tiling space. This can easily be derived from the results of Hadwiger (1940) (the same arguments can be found in Coxeter (1973)) or from a simple counterexample for $N=2, D=1$. Therefore, let us consider a 'preliminary' (arbitrary) embedding $E_{T}^{D} \subset E^{N}$ of the tiling space, and denote 
by $\bar{t}_{i}$ the corresponding tiling vectors. We define a linear transformation $\bar{A}: E^{N} \rightarrow$ $\tilde{E}_{T}^{D} \subset E^{N}$ by its action on the basis $\left\{b_{i}\right\}_{i-1, \ldots, N}$ :

$$
\tilde{A} b_{i}=\tilde{t}_{i} \quad i=1, \ldots, N \text {. }
$$

Since the orthogonal complement of the kernel of $\tilde{A}$, (ker $\tilde{A})^{\perp}$, and the preliminary tiling space $\tilde{E}_{T}^{\mathrm{D}}$ have the same dimension, there exists an orthogonal transformation $R: E^{N} \rightarrow E^{N}$, which transforms $\tilde{E}_{T}^{D}$ onto $(\operatorname{ker} \tilde{A})^{\perp}$. The action of $R$ on the orthogonal complement of $\tilde{E}_{T}^{D}$ is irrelevant for our purposes. The "final' tiling space is now identified with $(\operatorname{ker} \tilde{A})^{\perp}, E_{T}^{D} \equiv(\operatorname{ker} \tilde{A})^{\perp}$. The linear transformation $A \equiv R \circ \tilde{A}$ maps the lattice hypersurface $\Sigma$ onto $E_{T}^{\mathrm{P}}$. In particular, the tiling vectors are given by

$$
t_{i}=A b_{i} \equiv R \circ \tilde{A} b_{i} \quad i=1, \ldots, N .
$$

Since the kernels of $A$ and $\tilde{A}$ are the same, $E_{T}^{D}$ and $\operatorname{ker} A$ are orthogonal. Hence, $A$ has the property

$$
A=A \circ P=\left(A\left\lceil E_{T}^{D}\right) \circ P\right.
$$

where $P$ is the orthogonal projection onto $E_{T}^{D}$ and $A \mid E_{T}^{D}$ denotes the restriction of $A$ to $E_{T}^{D}$. Therefore, we can identify

$$
T \equiv A \mid E_{T}^{D}
$$

which completes the proof that the two generalised methods are equivalent.

We close this section with some remarks.

(i) The independence of the grid vectors and the tiling vectors allows us to fix the topology of the tiling and the shapes of the tiles independently from each other. In the projection formalism, this corresponds to the freedom of choosing the grid space and the tiling space independently.

(ii) The lattice $\mathscr{L}$ in $E^{N}$ is not uniquely specified by the grid. One may ask therefore whether, given a grid and a set of tiling vectors $t_{\mathrm{in}}$ there exists a special choice for the lattice, for which $E_{T}^{D}$ can be embedded in such a way that the $t_{1}$ are just orthogonal projections of the lattice vectors $b_{i}$ i.e. $T=1$. It is easy to find examples in the simple case $D=1, N=2$, where this is impossible. However, we do not know the general conditions one has to impose on the grid and the tiling vectors in order to reduce $A$ to a bare projection $(T=1)$.

(iii) The question whether or not the tiling has any translational symmetry can easily be answered within the projection formalism. Since the tiling is a linear transformation of some subset of the strip $S$, it has a translational symmetry if and only if this is the case for $S$. This in turn is true if and only if the grid space $E_{T}^{D}$ contains a lattice vector $u$. In formal terms, every lattice vector $u \in L$ contained in $E_{G}^{D}$ leads to a translational symmetry $A u$ of the tiling (for examples see \$8).

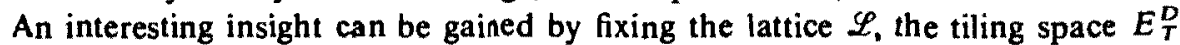
and the transformation $T$, but allowing the grid space $E_{T}^{D}$ to vary. By yarying $E_{G}^{D}$ we change the topology of the tiling, while the shapes of the tiles are kept fixed. If we keep the change in the orientation of $E_{G}^{D}$ small enough, an arbitrary large portion of the tiling containing the origin is left unchanged. In particular, since there are always large lattice vectors almost parallel to $E_{G}^{D}$, by a slight change of $E_{G}^{D}$ we can turn a non-periodic tiling into a periodic one with a large unit cell, keeping fixed a considerable portion around the origin. Hence, by looking at a finite piece of a tiling, we can never decide whether it is part of a truly non-periodic tiling or of a periodic one with a large unit cell. 
(iv) Further generalisations of the grid method exist. The hyperplanes of the grid $G_{N}^{D}$ need not be arranged periodically; the grid method, with a suitably defined vector function $K(y)$, still yields perfect tilings. The correspondence to the projection framework is somewhat more complicated, however, and we do not discuss it here. We just stress that the tiling cannot be obtained by simply arranging the hyperplanes of the lattice $\mathscr{L}$ aperiodically. The aperiodic lattice, when transformed by $A$, would lead to a pattern consisting of tiles whose shapes depend on their location. This is obviously not the case for the tiles generated by the grid method, whose shapes are defined by fixed tiling vectors.

\section{Fourier transformation}

The particular properties (13) and (14) of the linear transformation $A$ make it easy to apply the recently developed methods for the evaluation of Fourier spectra. $A$ is the product of an orthogonal projection $P:(S \cap L) \rightarrow E_{T}^{D}$ and a linear transformation $T: E_{T}^{D} \rightarrow E_{\tau}^{D}$. We therefore subdivide the calculation into lwo steps. We first determine the Fourier transform of the projected pattern and then include the effect of the transformation $T$.

For the following, it is convenient to use the orthogonal decomposition $E^{N}=E_{\tau}^{D} \oplus$ $E_{T, \perp}^{D}$. For each $x \in E^{N}$, we write $x=x_{\|}+x_{\perp}$, where $x_{\|} \in E_{T}^{D}$ and $x_{\perp} \in E_{T, \perp}^{D}$. In order to make the notation simple, we use the density of lattice points,

$$
\rho_{L}(x)=\sum_{j \in Z^{N}} \delta\left(x-\sum_{i=1}^{N} j_{i} b_{l}\right)
$$

instead of the lattice $L$ itself (see definition (5)). Following Zia and Dallas (1985), we write the projected density as

$$
\begin{aligned}
Q\left(x_{\|}\right) & =\int \mathrm{d}^{N-D} x_{\perp} C_{S}\left(x_{\|}, x_{\perp}\right) \cdot \rho_{L}\left(x_{\|}, x_{\perp}\right) \\
& =\int \mathrm{d}^{N-D} k_{\perp}^{\prime} \tilde{C}_{S}\left(x_{\|},-k_{\perp}^{\prime}\right) \cdot \rho_{L}\left(x_{\|}, k_{\perp}^{\prime}\right)
\end{aligned}
$$

where $C_{s}\left(x_{\|}, x_{1}\right)$ is the characteristic function of the strip $S$, and the tilde denotes the Fourier transform with respect to $x_{\perp}$. In contrast to the case of Zia and Dallas (1985), $C_{S}$ is $x_{1}$ dependent because $E_{T}^{D}$ and $S$ are not parallel here. The Fourier transform $\hat{Q}\left(k_{\mathbb{l}}\right)$ of $Q\left(x_{\mathfrak{q}}\right)$ is just a convolution:

$$
\begin{aligned}
& \hat{Q}\left(k_{\mathbb{1}}\right)=\int \mathrm{d}^{D} k_{\|}^{\prime} \int \mathrm{d}^{(N-D)} k_{\perp}^{\prime} V_{S}\left(k_{\|}-k_{1,}^{\prime}-k_{\perp}^{\prime}\right) \cdot \tilde{\rho}_{L}\left(k_{\eta}^{\prime}, k_{\perp}^{\prime}\right) \\
& =\int \mathrm{d}^{N} k^{\prime} V_{s}\left(k_{\|}-k_{\natural}^{\prime},-k_{\perp}^{\prime}\right) \cdot \rho_{L}\left(k^{\prime}\right)
\end{aligned}
$$

where $k^{\prime}=k_{1}^{\prime}+k_{\perp}^{\prime} . V_{S}$ is the Fourier transform of $C_{S}$ and $\rho_{C}(k)$ the Fourier transform of $\rho_{L}(x)$, i.e. the density of points in the reciprocal lattice $\bar{L}$. Therefore $\hat{Q}\left(k_{k}\right)$ is a weighted sum of $\delta$ functions. 
Now the transformation $T$ can easily be incorporated. We denote the transformed density by $Q_{r}\left(x_{1}\right)$; after some algebra, we find

$$
Q_{r}\left(x_{1}\right)=\frac{1}{|\operatorname{det} T|} Q\left(T^{-1} x_{\sharp}\right)
$$

and

$$
\hat{Q}_{r}\left(k_{\sharp}\right)=\hat{Q}\left(T^{+} k_{\sharp}\right)
$$

where $T^{+}$is the adjoint of $T$. Inserting this result into (15), we get the Fourier transform $\mathscr{F}\left(\boldsymbol{k}_{\|}\right)$of the final pattern:

$$
\mathscr{F}\left(k_{\|}\right) \equiv \hat{Q}\left(T^{+} k_{\mathbb{1}}\right)=\int d^{N} k^{\prime} V_{s}\left(T^{+} k_{\|}-k_{\|}^{\prime}-k_{1}^{\prime}\right) \cdot \rho_{L}\left(k^{\prime}\right) .
$$

\section{Symmetric two-dimensional grids}

In this section, we discuss the special class of $2 \mathrm{D}$ tilings generated by grids whose grid vectors $\left\{g_{1}\right\}_{i-1, \ldots . N}$ form an $N$-fold symmetric star, i.e. point to the vertices of a regular $N$-gon. The tiling vectors $t_{i}$ are assumed to have the same directions as the grid vectors. For later convenience, we choose the normalisations

$$
g_{1}=(N / 2)^{1 / 2} e_{i} \quad t_{i}=(2 / N)^{1 / 2} e_{i}
$$

(with the unit vectors $e_{i}$ forming an $N$-fold symmetric star).

We now describe how to obtain these tilings by the projection method. As it turns out, for our choice of grid and tiling vectors it is possible to use a cubic lattice

$$
\mathscr{L}=\left\{x \in E^{N} \mid(x-\gamma) \cdot h_{l}=k_{1} ; k_{1}=1, \ldots, N, l \in \mathbb{Z}\right\}
$$

where the components of $\gamma$ are the grid parameters $\gamma_{i}$ and $\left\{h_{i}\right\}_{1-1, \ldots, N}$ is an orthonormal basis of $E^{N}$. The high symmetry of the problem allows the application of power[ul group theoretic methods. The point group of $\mathscr{L}$ is the hyperoctahedral group $\Omega(N)$. Its natural representation $D^{N}$ is reducible under subduction to the subgroup $C_{N} \subset$ $\Omega(N)$ of all cyclic permutations of the basis vectors of the lattice. Its decomposition depends on whether $N$ is even or odd:

$$
D^{N} \underset{\Omega(N) \rightarrow C_{N}}{\longrightarrow} \begin{cases}D_{i}^{\prime} \oplus \sum_{i=1}^{[N / 2]} D_{i}^{2} & N \text { odd } \\ D_{i}^{1} \oplus D_{n / 2}^{1} \oplus \sum_{i=1}^{N / 2-1} D_{i}^{2} & N \text { even. }\end{cases}
$$

(The upper index denotes the dimension of the representations; $[n / 2]$ is the largest integer smaller than or equal to $n / 2$.) The invariant subspace $U^{2}$ of one of the $2 \mathrm{D}$ representations is spanned by the basis

$v_{1}=\left(\frac{2}{N}\right)^{1 / 2} \sum_{i=1}^{N} h_{l} \cdot \cos \left(\frac{2 \pi}{N}(l-1)\right) \quad v_{2}=\left(\frac{2}{N}\right)^{1 / 2} \sum_{l=1}^{N} h_{l} \cdot \sin \left(\frac{2 \pi}{N}(l-1)\right)$.

Starting from de Bruijn's arguments for the special case $N=S$ it is straightforward to show that the grid construction corresponds to the orthogonal projection $P$ of $(L \cap S)$ onto $U^{2}$ (here $S$ is the strip around $U^{2}$ defined by (6) and (22)). It is easy to show that the grid and tiling vectors that correspond to our projection construction are 
indeed given by (19). In the language of the generalised grid method we have therefore

$$
\begin{aligned}
& A=P \quad \text { i.e. } T=1 \\
& E_{T}^{2}=E_{G}^{2}=U^{2} .
\end{aligned}
$$

From remark (iii) in $\S 6$, it follows that the tilings have a translational symmetry $P u$ if there exists a lattice vector $u \in L$ and real numbers $r$ and $s$ such that

$u=\left(\frac{N}{2}\right)^{1 / 2}\left(r v_{1}+s v_{2}\right)=\sum_{l=1}^{N} h_{1} \cdot\left[r \cos \left(\frac{2 \pi}{N}(l-1)\right)+s \sin \left(\frac{2 \pi}{N}(l-1)\right)\right]$.

The condition that $u \in U^{2}$ is a lattice vector means that

$$
r \cos \left(\frac{2 \pi}{N}(l-1)\right)+s \sin \left(\frac{2 \pi}{N}(l-1)\right) \in Z \forall L
$$

It is easy to see that this can only be satisfied for $N=2,3,4,6$, which are just the allowed crystal symmetries.

Obviously, the generalised grid method allows the construction of 2D quasiperiodic patterns with any point symmetry. This is in contrast to the results of Levine and Steinhardt (1984). Using a different construction, they get, besides the allowed crystal symmetries, a rather restricted set of possible point symmetries $(N=8, P$ or $2 P$, where $P$ is a prime number). The difference may be due to additional conditions imposed by Levine and Steinhardt, e.g. self-similarity.

The number $M_{N}$ of different tiles (where we do not distinguish between different orientations) of a tiling generated by a symmetric $N$ grid and a symmetric star of tiling vectors is easily found to be

$$
M_{N}= \begin{cases}{[N / 2]} & N \text { odd } \\ {[N / 4]} & N \text { even }\end{cases}
$$

where again $[x]$ is the largest integer smaller or equal to $x$ Interestingly enough, $M_{N}$ coincides with the number of incommensurate intervals Levine and Steinhardt (1984) need in their alternative construction of quasiperiodic lattices.

In figure 2 we present a tiling exhibiting 12-fold symmetry. Its tiles are a square and two rhombuses, one with $60^{\circ}$ and one with a $30^{\circ}$ angle. This tiling looks strikingly similar to electron microscope images of a new phase of $\mathrm{Ni}-\mathrm{Cr}$ alloy recently discovered (Ishimasa et al 1985).

\section{Quasiperiodic tilings with periodic inclusions}

As a final application we discuss a new principle for the generation of quasiperiodic patterns containing arbitrarily large periodic regions. We illustrate this principle with a simple example. Take, e.g. a 5-grid, in which two arrays of grid lines are narrowed with respect to the remaining ones, as shown in figure $3(a)$. We observe that

(i) to all intersection points of two given grid arrays, there corresponds a unique rhombus having a unique orientation;

(ii) the rhombuses corresponding to two adjacent intersection points have one common edge (by 'adjacent', we mean that the two intersection points are not separated by any grid line). 
Therefore the intersection points lying in a region bounded by thin grid lines (see figure $3(a)$ ) give rise to rhombuses that are arranged periodically. Note, however, that 5 -fold symmetry is destroyed by this procedure. An example is shown in figure $3(b)$.

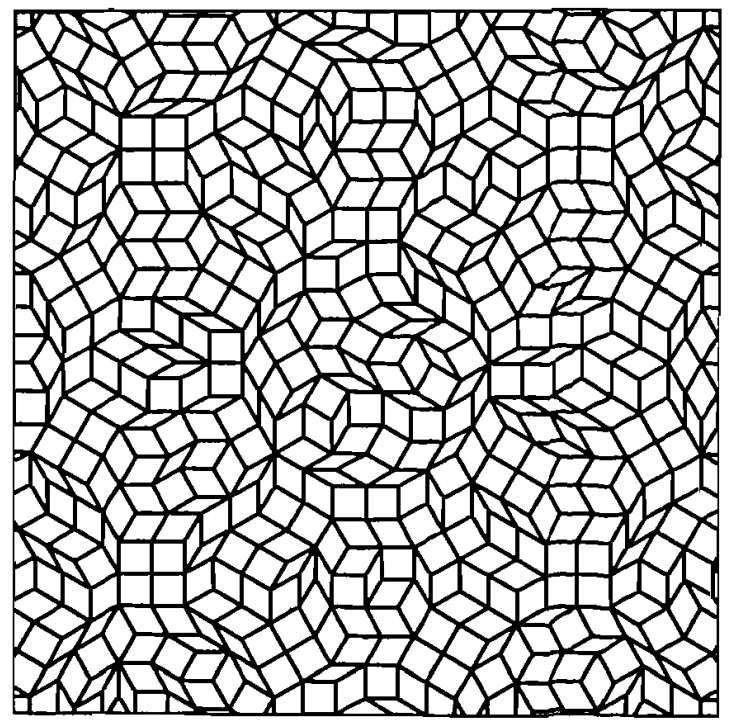

Figure 2. Pattern with 12-fold bond orientational symmetry. Its Fourier spectrum consists of a 12-fold symmetric arrangement of $\delta$ peaks filling reciprocal space densely.

(a)

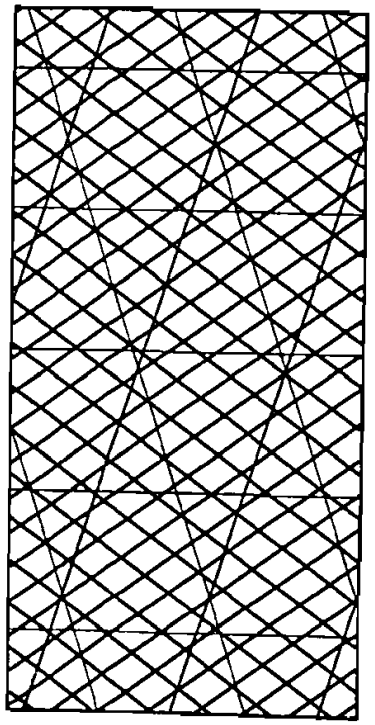

(b)

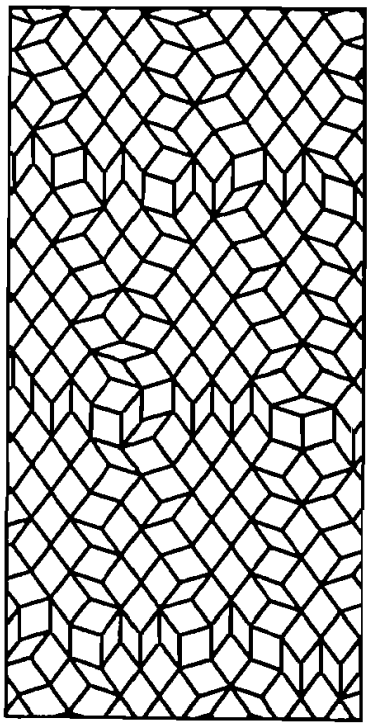

Figure 3. (a) Example of a grid yielding periodic inclusions. The areas bounded by the thin grid lines generally contain a lot of intersection points of the same type, leading to adjacent tiles of the same type. (b) Tiling with periodic inclusions. The tiles are the Penrose rhombuses. 


\section{Acknowledgments}

The authors wish to thank W Baltensperger, G Blatter, J Fröhlich and T M Rice for encouragement and helpful discussions, as well as $R$ Schilling for sending a copy of the preprint of Socolar et al. One of us (JR) thanks the Schweizerischer Nationalfonds for financial support.

Note added. Towards the end of the preparation of this paper we received a very interesting preprint from Socolar el al (1985). They also consider generalisations of de Bruijn's method. The connection to the projection formalism is not discussed however.

\section{References}

Coxeter H S M 1973 Regular Polytopes (New York: Dover) p $250 \mathrm{ff}$ de Bruijn N G 1981 Ned. Akad. Wetensch Proc. Ser. A 43 39-66

Duneau $M$ and Katz A 1985 Phys. Reu. Lett. 54 2688-91

Elser V 1985 AT T Bell Lab Preprint

Gasdner M 1977 Sci Am (Jan) 110-21

Hadwiger H 1940 Comen. Math. Helv. 1390-107

Ishimasa T. Nissen H U and Fukano Y 1985 Phys Reu. Lelt. $35511-3$

Kalugin P A, Kitaev A Yu and Levitov LS 1985 Zk. Eksp. Theor. Fiz Pis. Red. 11 119-21 (Engl. transl. JETP LetL 41 145-9)

Kramer P and Neri R 1984 Acta Crystallogr. A $40580-7$

Levine D and Steinhardt P J 1984 Phys. Rev. Lett. $532477-80$

Mackay A L 1981 Sou. Phys.-Crystallogr. 26 517-22

Penrose R 1979 Math, Intell 2 32-7

Shechtman D. Blech I. Gratias D and Cahn J W 1984 Phys. Reu Let. 53 1951-3

Socolar J, Levine D and Steinhardt PJ 1985 University of Penusyluania Preprint

Zia R K P and Dallas W J 1985 J. Phys. A; Math. Gen. 18 L31-S 